\title{
ANALYSIS OF BEPCII OPTICS USING ORBIT RESPONSE MATRIX *
}

\author{
Y.Y. Wei, IHEP, Beijing 100049, P.R.China
}

\section{Abstract}

Due to the errors in all kinds of components of storage ring, the real ring optics is different from the design one. A computer code LOCO was developed at SLAC to calibrate the linear optics based on the closed orbit response matrix. This paper discusses mainly on the procedure and results of optics correction at BEPCII BPR. Using LOCO, we determined the errors of quadrupole strengths, BPM gains and corrector kicks, and found the quadrupole strengths that best restore the design optics with sextupoles on. Optics measurement after correction shows the real optics agrees well with the design one.

\section{INTRODUCTION}

BEPCII is an upgrade project of the Beijing ElectronPositron Collider (BEPC), in which a new inner ring was installed inside the old one. It is constructed for both high energy physics and synchrotron radiation (SR) experiments. The storage rings for collision consist of a positron and an electron ring. The two beams collide at the south IP with a crossing angle of $11 \mathrm{mrad} \times 2$, and the luminosity is optimized to $1 \times 10^{33} \mathrm{~cm}^{-2} \mathrm{~s}^{-1}$ at $1.89 \mathrm{GeV}$. The outer parts of two rings contribute to the SR ring, in which the beam current is $250 \mathrm{~mA}$ at $2.5 \mathrm{GeV}$.

Due to the limited space and the consideration for both collision and SR, the geometric and optics designs of the BEPCII are relatively complicated, and the 4-fold symmetric structure of the BEPC does no exist. The arc region consists of 6 quasi-FODO cells with the fifth and eleventh bending magnets missing. The quadrupoles and sextupoles of arc regions are installed very closely and there is no special region for dispersion suppressing. In the IR region, the superconducting quadrupole SCQ not only squeezes the vertical beta function but also bends the beam further, and 4 warm bore quadrupoles are used for connecting the arc and IP. In the RF region, there is no symmetry in the inner and outer rings. Because of all this, a good agreement between the real optics and the model is essential for the BEPCII to achieve the maximum performance.

During the commissioning of the BEPCII, which started from Nov. 2006, we used the Linear Optics from Closed Orbits (LOCO) to correct the optics successfully. In this paper, we present the results of our fist attempt to use LOCO at the BEPCII, including a brief introduction of the LOCO algorithm, the analysis and optics measurement after correction on the positron ring (BPR). For the electron ring (BER), the procedure and results are similar, and omitted here.

\footnotetext{
*Work supported by Core University Program of CAS-JSPS

\#weiyy@mail.ihep.ac.cn
}

\section{BASIC THEORY}

The orbit response matrix is defined as:

$$
\left(\begin{array}{l}
\Delta x \\
\Delta y
\end{array}\right)=M\left(\begin{array}{l}
\Delta \theta_{x} \\
\Delta \theta_{y}
\end{array}\right)
$$

where $\Delta \theta_{x}$ and $\Delta \theta_{y}$ are the changes in strength of horizontal and vertical correctors respectively, $\Delta x$ and $\Delta y$ are the orbit perturbations at the BPMs. $M$ is an $m \times n$ matrix, where $m$ is the number of BPMs, and $n$ is the number of horizontal and vertical correctors. First, a response matrix $M_{\text {meas }}$ is measured. Then, the accelerator modelling code such as AT [1] is used to calculate the model matrix $M_{\text {model }}$. By varying the quadrupole strengths, corrector kicks and BPM gains in model, we can minimize the difference between the measured and model response matrices as [2]:

$$
\chi^{2}=\sum_{i, j} \frac{\left(M_{\text {mod }, i j}-M_{\text {meas }, i j}\right)^{2}}{\sigma_{i}^{2}} \equiv \sum_{i, j} V_{i j}^{2},
$$

where $\sigma_{i}$ is the measured noise level for BPMs, $V_{i j}$ is the function of the parameters varied in the model lattice and can by expressed by[3]:

$$
\Delta V_{i j}=\sum \frac{\partial V_{i j}}{\partial K_{q}} \Delta K_{q}+\sum \frac{\partial V_{i j}}{\partial G_{i}} \Delta G_{i}+\sum \frac{\partial V_{i j}}{\partial \theta_{j}} \Delta \theta_{j}+\sum \frac{\partial V_{i j}}{\partial \delta_{j}} \Delta \delta_{j}+\ldots . .
$$

where $\Delta K_{q}, \Delta G_{i}, \Delta \theta_{j}$ are the changes in quadrupole strengths, BPM gains and corrector kicks, respectively. The $\Delta \delta_{j}$ is the energy shift induced by the change of the $j^{\text {th }}$ corrector strength, which locates in a dispersion region.

In using LOCO, with the measured data and the initial parameters we calculate the initial $V_{i j}$ first, solving the change of all the parameters from equation (3) by singular value decomposition to minimize $\sum_{i j}\left(V_{i j}+\Delta V_{i j}\right)^{2}$, modifying the parameters and repeating above procedure until the residual errors of $M_{\text {meas }}$ and $M_{\text {model }}$ converge to the noise level of BPM. With the parameters derived from LOCO, the model optics can predict the real one, and after the parameters are corrected, the design optics can be restored.

\section{MEASUREMENT}

In each of the BEPCII storage rings, 34 horizontal correctors, including 30 trim coils of bending magnets and 4 correctors, 33 vertical correctors and 64 doubleview BPMs are available. This results in $(34+33) \times 64 \times 2=$ 8576 elements that can be used for fitting in model optics.

Before the orbit response data were collected, we measured the BPM offsets using beam-based alignment, and the orbit was corrected to the centre of quadrupoles. The orbit after correction is within $\pm 2 \mathrm{~mm}$ in horizontal and $\pm 1 \mathrm{~mm}$ in vertical. 
Measurement is done automatically, with corrector strengths changed one by one in bi-directional way, and the orbit shift is taken averaging over 10 times data to reduce the random error due to BPM resolution. It takes us nearly two and a half hours to complete the measurement once. The kick size of corrector is $0.2 \mathrm{mrad}$, and the rms orbit perturbation is about $1 \mathrm{~mm}$. At the same time, the BPM resolution is also measured and served as weight in LOCO fitting.

The measured horizontal and vertical dispersion are necessary for LOCO, considering the dispersion correction and help to break the degeneracy between corrector strengths and BPM gains.

\section{ANALYSIS ON POSITRON RING}

During the commissioning of the BEPCII, we restored the optics of BPR and BER by analysing the response matrices. Here, we only present the procedure and results of BPR in detail.

\section{Measurement with sextupoles off}

Because of the feed down effect of orbit errors in sextupoles, we first measured the response matrix with sextupoles off, and the parameters listed below were varied in fitting the model to the measured response matrix:

- 128 BPM gains and couplings.

- 67 corrector magnet kicks and couplings;

- 67 energy shifts at the correctors;

- 70 strengths of quadrupoles;

The comparison of measured response matrix and difference between the measured and fitted model response matrix are shown in figure 1 . Note that after fitting, the residual orbit is much smaller.

The distribution of residuals for the response matrix is shown in Fig.2. The distribution has a width roughly equal to 1 , which indicates the fitting in LOCO converged to the noise level of BPM. In fact, the rms error of the measured and fitted response matrix after fitting is about
$0.007 \mathrm{~mm}$, except for some abnormal data removal, and the measured BPM resolution is smaller than $0.01 \mathrm{~mm}$.

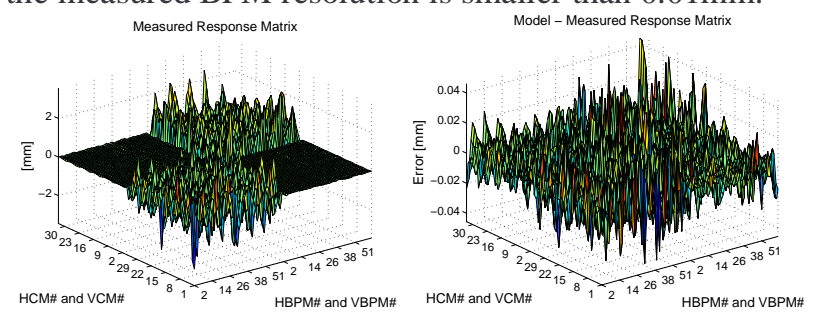

Figure 1: Measured response matrix (left) and difference between measured and model fitted by LOCO (right)

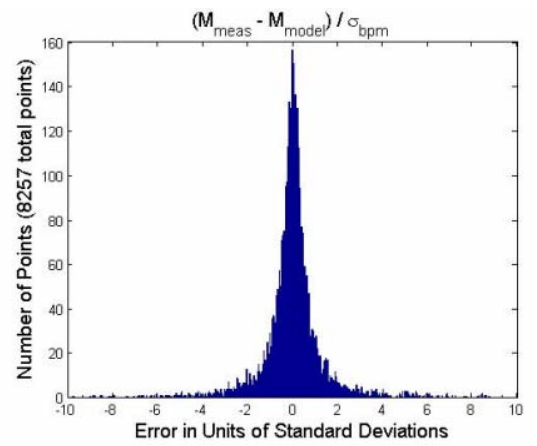

Figure 2: Distribution of residual differences between measured and fitted orbit response matrices, normalized to the noise level of the respective BPMs

Fig. 3 shows the errors of quadrupole fudge factors. The quadrupole amplitude fudge factor $\mathrm{AF}$ represents the relative difference between the strength found by LOCO and the magnet strength in a nominal design model:

$$
A F^{i}=K_{1}^{i} / K_{L}^{i} \text {. }
$$

With eq. (4), we can calculate the fudge factor of the $i^{\text {th }}$ quadrupole. Here, $K_{L}$ is the strength determined by LOCO, and $K_{l}$ is the nominal strength. Then, the strength $K_{C}$ that can best restore the design optics derives from

$$
K_{C}^{i}=K_{1}^{i} A F^{i} \text {. }
$$

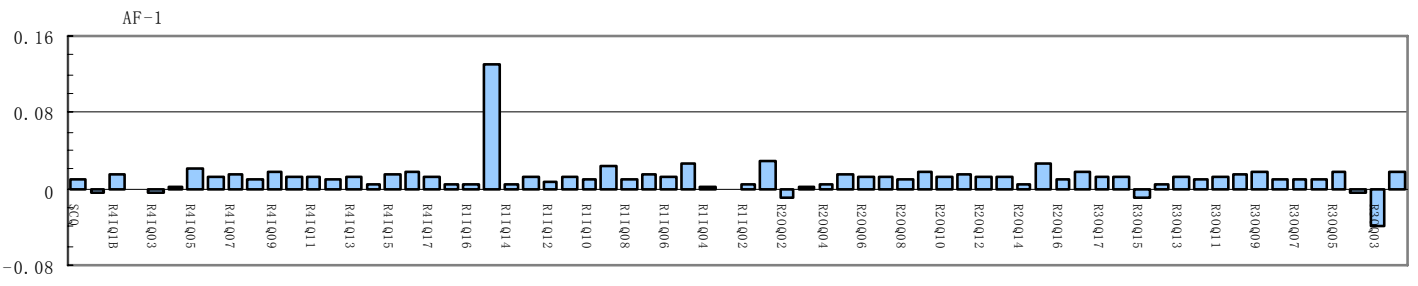

Figure 3: Errors of quadrupole fudge factors ( sextupoles off)

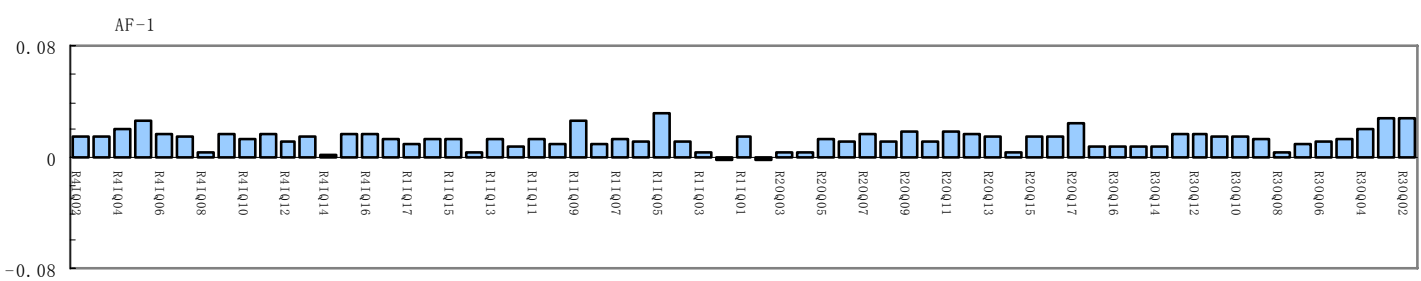

Figure 4: Errors of quadrupole fudge factors ( sextupoles on) 
We can note from Fig. 3 that two adjacent quadrupoles with same polarity and no BPM between them are shown have large strength errors and sometimes fight each other. For example, Q15 and Q16, Q02 and Q03 are couples of that kind, and the strength error of R1IQ15 can be $13 \%$. R3OQ02's error is about 2\%, its adjacent magnet RQ3OQ03 is -3\%. It seems there is a problem in fitting for this case.

\section{Measurement with sextupoles on}

After analysing the strength errors of all quadrupoles, we attempt to compensate the optics with sextupoles on only tuning the strengths of quadrupoles. This time, we measured the response matrix with sextupoles on and LOCO setup included:

- Do not fit BPM gains, corrector kicks and their couplings

- Fit dispersion function

- Fit strengths of quadrupoles except SCQ, Q1A and Q1B. SCQ is a dipole-quadrupole combined-function magnet. Q1A and Q1B have the same power supplies with BER's.

- Replace the strengths of two adjacent quadrupoles with same polarity by one parameter fitted in LOCO.

The errors of quadrupole fudge factors that compensate the optics with sextupoles on are shown in Fig. 4. By tuning the quadrupole strengths, most optics of real machine were restored.

\section{OPTICS MEASUREMENT}

After the quadrupole strengths determined by LOCO were applied to the BPR of BEPCII, optics measurement was performed to examine the difference between the real and the design optics.

The nominal tune of the design optics was 6.54/5.59, and measured tune after correction was 6.539/5.589, which agreed well with the design tune.
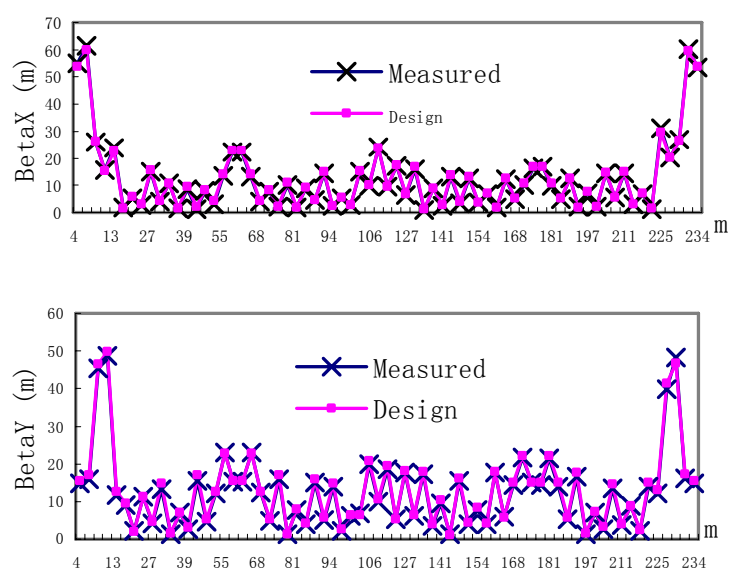

Figure 5: The comparison of the measured and design horizontal (top) and vertical (bottom) beta function.

Beta function of the whole ring was measured using quadrupole modulation method. Fig. 5 shows the comparison of the measured and design beta function.
We also measured the dispersion by changing the RF frequency. Fig. 6 indicates after the optics correction the distortion of dispersion is much smaller. Some places have extremely large errors in both horizontal and vertical planes because of the abnormal BPMs there.

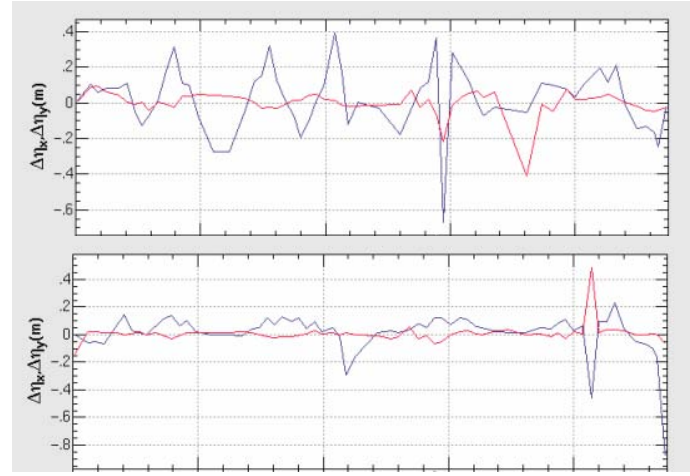

Figure 6: The distortion of dispersion before (top) and after (bottom) optics correction.

\section{CONCLUSION}

Analysis of the orbit response matrix determined the quadrupole strength errors, BPM gains, corrector kicks and their couplings for both BPR and BER. The fudge factors of quadrupole are mostly within 1.01 1.02, that means the real quadrupole strengths are lower than the design strengths. It can be explained by the effect of adjacent sextupole due to the short distance between them The fitted model accurately predicted the tune, beta and dispersion functions. The analysis also gave the best settings for quadrupoles to restore the design optics with sextupoles on. After correction, the measured beta function of BPR and BER at most quadrupoles can be restored within $\pm 10 \%$ of design model, except some places where the design beta functions are small and have the relatively large discrepancies due to the measurement accuracy. The distortion of dispersion function was decreased. With the successful optics correction, the commissioning of the BEPCII has made a good progress.

\section{ACKNOWLEDGEMENTS}

The programme used to collect the data and measure the optics is coded with SAD script from KEKB, and the author would like to thank all the participators work on BEPCII commissioning and J. Safranek for assistance with LOCO.

\section{REFERENCES}

[1] http://www-ssrl.slac.stanfor.edu/at/

[2] J. Safranek, "Experimental determination of storage ring optics using orbit response measurements", Mucl. Inst. And Meth. A388, 27 (1997).

[3] J. Safranek, "Calibration of the X-Ray Ring Quadrupoles, BPM, and Orbit Correctors Using the Measured Orbit Response Matrix", SLAC-PUB-6442, Feb. 1994 (A). 